\title{
Magnetic resonance imaging after most common form of concussion
}

\author{
Harald Schrader*1, Dalia Mickevičiene², Rymante Gleizniene ${ }^{3}$, \\ Silvija Jakstiene ${ }^{3}$, Danguole Surkiene ${ }^{2}$, Lars Jacob Stovner ${ }^{1}$ and \\ Diana Obelieniene ${ }^{2}$
}

\author{
Address: ${ }^{1}$ Department of Neurology and Clinical Neurophysiology, Trondheim University Hospital, 7006 Trondheim, Norway, ${ }^{2}$ Department of \\ Neurology, Kaunas University of Medicine, Kaunas, Lithuania and ${ }^{3}$ Department of Radiology, Kaunas University of Medicine, Kaunas, Lithuania \\ Email: Harald Schrader* - harald.schrader@ntnu.no; Dalia Mickevičiene - daliamickeviciene@gmail.com; \\ Rymante Gleizniene - rymangleiz@gmail.com; Silvija Jakstiene - sylvijajakstiene@zebra.lt; Danguole Surkiene - surkiene@gmail.com; \\ Lars Jacob Stovner - lars.stovner@ntnu.no; Diana Obelieniene - dianobel@takas.lt \\ * Corresponding author
}

Published: 17 June 2009

BMC Medical Imaging 2009, 9:II doi:I0.1 I86/1471-2342-9-II
Received: 31 October 2008

Accepted: 17 June 2009

This article is available from: http://www.biomedcentral.com/I47I-2342/9/II

(C) 2009 Schrader et al; licensee BioMed Central Ltd.

This is an Open Access article distributed under the terms of the Creative Commons Attribution License (http://creativecommons.org/licenses/by/2.0), which permits unrestricted use, distribution, and reproduction in any medium, provided the original work is properly cited.

\begin{abstract}
Background: Until now there is a lack of carefully controlled studies with conventional MR imaging performed exclusively in concussion with short lasting loss of consciousness (LOC).

Methods: A MR investigation was performed within 24 hours and after 3 months in 20 patients who had suffered a concussion with a verified loss of consciousness of maximally 5 minutes. As a control group, 20 age- and gender matched patients with minor orthopaedic injuries had a MR investigation using the same protocol.

Results: In a concussion population with an average LOC duration of I. 4 minutes no case with unequivocal intracranial traumatic pathology was detected.

Conclusion: An ordinary concussion with short lasting LOC does not or only seldom result in a degree of diffuse axonal injury (DAI) that is visualized by conventional MR with field strength of $I .0$ Tesla (T). Analysis of earlier MR studies in concussion using field strength of I.5 T as well as of studies with diffusion tensor MR imaging (MR DTI) reveal methodological shortcomings, in particular use of inadequate control groups. There is, therefore, a need for carefully controlled studies using MR of higher field strength and/or studies with MR DTI exclusively in common concussion with LOC of maximally 5 minutes.
\end{abstract}

\section{Background}

After concussion (or mild traumatic brain injury (MTBI)), a significant proportion of subjects report persisting symptoms that include headache, cognitive dysfunction, dizziness, fatigue, and irritability. This cluster of rather non-specific symptoms has been termed as the postconcussion syndrome (PCS), a condition that has been debated since the end of the $19^{\text {th }}$ century and still remains controversial. Several authors suggest that the symptoms of postconcussion syndrome are due to structural abnormalities and/or organic cerebral dysfunction $[1,2]$. Others have assumed that the PCS is psychogenic in origin or have proposed a biopsychosocial model to explain its development into chronicity in some individuals $[3,4]$. 
For the concept of PCS being caused by an organic injury it seems necessary to assume that the concussion produces long-lasting morphological and/or functional lesions. This is, however, still equivocal, at least when using neuroimaging procedures such as cerebral computer tomography (CT) or magnetic resonance (MR) imaging. Studies using CT show lesions only in a small minority of cases, whereas conventional MR studies show pathology in the acute state in 10 to $57 \%$ of cases [5-9]. All these studies included patients that had loss of consciousness (LOC) of up to 1520 minutes. However, in the most common form of concussion, LOC lasts only from a few seconds to few minutes [10]. Therefore, we wanted with a rigidly controlled design to study whether there may be cases with visually detectable and unequivocal traumatic lesions in conventional MR even if one restricts the investigation to only include cases of concussion with LOC of less than 5 minutes.

\section{Methods \\ Study group}

Since traumatic white matter lesions may be confused with foci of non-specific high signal intensity that is associated with normal aging $[11,12]$, the study group was restricted to individuals aged 18 to 40 years. Twenty individuals with concussion and short-lasting LOC who attended the emergency unit at the Kaunas Medical University Hospital during the duty of the participating investigators were consecutively identified if eligible according to strict inclusion and exclusion criteria. By thorough examination of witnesses it was in all cases assured that the LOC did not exceed 5 minutes. Additionally, inquiry was made about presence and duration of retrograde amnesia, anterograde amnesia and confusion. No patient was included in whom a reliable witness report could not be obtained.

The patients had no other injuries than the concussion, except for small skin lesions, bruises and other insignificant injuries. Exclusion criteria were diabetes, hypertension, affective disorders, prior history of alcohol or drug abuse, prior history of psychiatric or neurological disorder, prior history of epilepsy or seizure associated with the concussion, earlier concussion and concussion due to assault. There were also no focal neurological signs and normal neurological status at admission except for amnesia and slight and transitory confusion.

\section{Control group}

As controls, individually sex- and age (plus/minus 3 years of age) matched subjects aged 18 to 40 years with the same exclusion criteria as the study group were taken from the records of the traumatology department by identifying patients with minor to moderate non-head injuries who had attended this department in a time period of maxi- mally 2 weeks after the concussion date of the traumatized matching patient. If there were several potential controls within this period, the patient was taken who concerning the time of the attendance to the clinic was nearest that of the head-traumatized individual.

\section{MR imaging}

After inclusion, the patients with concussion and nonhead traumatized controls underwent an MR investigation within 24 hours after the trauma and after 3 months using the same protocol each time. MR imaging was performed on a Gyroscan T10-NT 1.0 T (Philips Medical System). A scout sequence was used to align the subsequent scans. The matrix in all sequences was $205 \times 256$. Axial imaging plane and $5 \mathrm{~mm}$ slice thickness was used for all patients in all sequences. Three pulse sequences were included: T1W/SE (TR - 586 ms, TE - 14 ms; NSA 2); T2W/FLAIR (TR - $5000 \mathrm{~ms}$, TE - $80 \mathrm{~ms}$, TI - $1900 \mathrm{~ms}$, NSA 1); and to detect hemosiderin deposits T2W/FFE (TR - 713 ms, TE - 21 ms; flip angle 15.0, NSA 2). The MR covered the whole brain. The scan time was 15 minutes and the slice number 22 .

Final evaluation of MR was made by two neuroradiologists blinded for the diagnostic status of the individual.

The study was in compliance with the ethical principles for medical research involving human subjects according to the Helsinki Declaration and was approved by the Ethical committee of the Medical Faculty of the University of Kaunas. All patients gave written informed consent to participate.

\section{Results \\ Study group and control group}

Of the twenty patients in the study group 10 were females (mean age 22.8 years; range 18 to 32 years) and 10 males (mean age 26.5 years; range 18 to 37 years) (see table 1 ). The concussion was in 12 cases due to a car accident, in 6 cases due to fall, in one case due to collision with a moving machine and in one case due to sporting activity. Duration of LOC was on average 1.4 minutes (range: 0.5 - 5 minutes). Mean duration of retrograde amnesia was 1.4 minutes (range: 0 - 10 minutes), of anterograde amnesia 4.3 minutes (range: $0-20$ minutes) and of confusion 7.3 minutes (range: 0 - 30 minutes).

Of the controls, the 10 females had a mean age of 22.9 years (range 18 to 32 years) and the 10 males a mean age of 26.0 years (range 18 to 39 years) (see table 2 ). The nonhead injuries were in 11 cases contusions in the extremities or of the back, in 3 cases distorsions in the ankle or knee, in 3 cases skin lesions and moderate wounds in the remainder (see table 2 ). 
Table I: Clinical characteristics and cerebral MR after concussion

\begin{tabular}{|c|c|c|c|c|c|c|c|c|}
\hline Gender & Age & Mechanism & $\begin{array}{l}\text { Unconsciousn } \\
\text { ess (min) }\end{array}$ & $\begin{array}{c}\text { Retrograde } \\
\text { amnesia (min) }\end{array}$ & $\begin{array}{c}\text { Confusion } \\
\text { (min) }\end{array}$ & $\begin{array}{c}\text { Anterograde } \\
\text { amnesia (min) }\end{array}$ & $\begin{array}{l}\text { Cerebral MR } \\
\text { within } 24 \text { hours } \\
\text { after trauma }\end{array}$ & $\begin{array}{c}\text { Cerebral MR } 3 \\
\text { months after } \\
\text { trauma }\end{array}$ \\
\hline $\mathrm{F}$ & 18 & MVA & 2 & 5 & 15 & 0 & NP & NP \\
\hline $\mathrm{F}$ & 18 & Fall & 1 & 0 & 0 & 0 & NP & NP \\
\hline $\mathrm{F}$ & 19 & MVA & 0,5 & 0 & 10 & 5 & NP & NP \\
\hline $\mathrm{F}$ & 19 & MVA & 2 & 0 & 0 & 0 & NP & NP \\
\hline $\mathrm{F}$ & 20 & Sporting & I & 0 & 0 & 0 & NP & - \\
\hline $\mathrm{F}$ & 22 & MVA & 0,5 & 3 & 0 & 1,5 & NP & NP \\
\hline$F$ & 25 & Fall & 0,5 & 0 & 5 & 0 & $\begin{array}{l}\text { Subependymal } \\
\text { periventricular } \\
\text { heterotopia }\end{array}$ & Unchanged \\
\hline $\mathrm{F}$ & 27 & MVA & I & 0 & 0 & 3 & NP & NP \\
\hline $\mathrm{F}$ & 28 & MVA & 2 & 0 & 30 & 20 & NP & - \\
\hline $\mathrm{F}$ & 32 & Fall & 0,5 & 0 & 10 & 5 & NP & NP \\
\hline$M$ & 18 & MVA & 1 & 5 & 0 & 15 & NP & NP \\
\hline$M$ & 18 & MVA & I & 0 & 0 & 0 & NP & NP \\
\hline$M$ & 21 & MVA & i & 0 & 15 & 5 & NP & NP. \\
\hline$M$ & 21 & Fall & 3 & 0 & 0 & 0 & $\begin{array}{c}\text { Nonspecific } \\
\text { subcortical focus } \\
\text { in left frontal } \\
\text { region }\end{array}$ & Unchanged \\
\hline$M$ & 26 & Moving machine & I & 0 & 10 & 5 & NP & NP \\
\hline$M$ & 29 & Fall & I & 0 & 0 & 0 & $\begin{array}{c}\text { Few nonspecific } \\
\text { subcortical } \\
\text { hyperintensive } \\
\text { T2W/FLAIR foci } \\
\text { in frontal regions }\end{array}$ & Unchanged \\
\hline M & 31 & Fall & 1 & 0 & 5 & 2 & $\begin{array}{c}\text { Ependymal cyst in } \\
\text { left lateral } \\
\text { ventricle }\end{array}$ & Unchanged \\
\hline$M$ & 32 & MVA & 5 & 0 & 10 & 10 & $\begin{array}{c}\text { Multiple } \\
\text { nonspecific } \\
\text { subcortical } \\
\text { hyperintensive } \\
\text { T2W/FLAIR foci } \\
\text { in both fronto- } \\
\text { parietal regions }\end{array}$ & Unchanged \\
\hline$M$ & 32 & MVA & 3 & 5 & 5 & 0 & $\mathrm{NP}$ & NP \\
\hline$M$ & 37 & MVA & 0,5 & 10 & 30 & 15 & NP & NP \\
\hline
\end{tabular}

MVA: motor vehicle accident NP: no pathology

\section{MR imaging}

There was complete inter-rater agreement both for the study group and for the control group, possibly due to the low incidence of pathological findings. MR shortly after the concussion showed no intracranial pathology in 15 patients and was still without pathology after 3 months (see table 1). In particular, there were no visible signs of diffuse axonal injury or micro-hemorrhages. In two of these patients a second MR investigation after 3 months could not be performed due to technical problems. One patient had multiple unspecific high signal intensity foci in both fronto-parietal regions. Another patient had a few hyperintensive foci in both frontal regions, and a third patient a hyperintensive focus in the left frontal region. All three patients were male. The hyperintensive foci were unchanged both concerning number and size in the MR after 3 months. An incidental finding was an ependymal cyst in left lateral ventricle in one patient and a subependymal periventricular heterotopia in another patient.

MR in the control group shortly after the trauma and 3 months later showed no pathology in 18 patients (see table 2). One male control had a nonspecific hyperintensive focus in the left peritrigonal region and another control a pineal cyst.

The prevalence of cases with hyperintensive focus/foci of the control group ( $\mathrm{n}=1)$ was not significantly different from that in the study group $(n=3)(p=0.60$; two sided Fisher's exact test) 
Table 2: Cerebral MR after minor non-head injuries

\begin{tabular}{|c|c|c|c|c|}
\hline Gender & Age & Trauma & Cerebral MR shortly after trauma & Cerebral MR 3 months after trauma \\
\hline $\mathrm{F}$ & 18 & Elbow contusion & NP & NP \\
\hline $\mathrm{F}$ & 19 & Hand contusion & NP & NP \\
\hline $\mathrm{F}$ & 19 & Hand contusion & Pineal cyst & Unchanged \\
\hline $\mathrm{F}$ & 19 & Hand contusion & NP & NP \\
\hline $\mathrm{F}$ & 20 & Knee contusion & NP & NP \\
\hline $\mathrm{F}$ & 23 & Ankle distorsion & NP & NP \\
\hline $\mathrm{F}$ & 25 & Leg wound & NP & NP \\
\hline $\mathrm{F}$ & 26 & Scratch knee cap & NP & NP \\
\hline $\mathrm{F}$ & 28 & Knee contusion & NP & NP \\
\hline $\mathrm{F}$ & 32 & Finger cut & NP & NP \\
\hline$M$ & 18 & Abdominal wound & NP & NP \\
\hline$M$ & 19 & Leg contusion & NP & NP \\
\hline$M$ & 20 & Ankle contusion & NP & NP \\
\hline$M$ & 20 & Back contusion & NP & NP \\
\hline$M$ & 26 & Finger cut & NP & NP \\
\hline$M$ & 27 & Leg wound & $\begin{array}{c}\text { Nonspecific T2W/FLAIR hyperintensive focus in left peritrigonal } \\
\text { white matter }\end{array}$ & Unchanged \\
\hline$M$ & 30 & Ankle distorsion & NP & NP \\
\hline$M$ & 30 & Hand contusion & NP & NP \\
\hline$M$ & 31 & Ankle distorsion & NP & NP \\
\hline$M$ & 39 & Knee contusion & NP & NP \\
\hline
\end{tabular}

NP: no pathology

\section{Discussion}

In the present study in which the most common type of concussion was investigated by MR imaging and inclusion of age-and gender matched non-head injured controls, no case was detected with unequivocal intracranial traumatic pathology. There were three male subjects in which high signal intensity foci were found in the white matter. The changes had the same appearance 3 months post injury and were, therefore, likely to be pre-existing findings of non-traumatic origin. In the control group only one individual had a non-traumatic, pre-existing hyperintensive focus. Possibly due to the low power of the study, the difference in prevalence of pre-existing hyperintensive foci was insignificant. Nevertheless, this result may indicate that individuals prone to head injury differ from healthy individuals and individuals prone to orthopaedic injuries in respect to pre-traumatic MR findings.

The study had the limitation that it was performed with 1.0 T MR and $5 \mathrm{~mm}$ slices and that traumatic lesions such as small sub-cortical hemorrhages shown by high field MR may have escaped detection. On the other hand, diffuse axonal injury can be demonstrated with MR of even lower field strength than in the present study [13].

A general methodological problem for earlier studies on concussion with structural neurodiagnostic techniques performed in the last 25 year is that many of them did not have a control group and in those who had, the control group was mostly inadequate. An adequate control group should be age- and gender-matched individuals with minor non-brain injuries such as in the present study. Presumably, such group would have a socio-economic status as well as a degree of accident-proneness, psychopathology and alcohol consumption that is more similar to individuals with concussion than a control group of healthy individuals. It is known that psychopathology may predispose to injuries $[14,15]$ and there are more pathological MR findings in people with psychopathology than in healthy individuals [16-18]. Due to the lack of an adequate control group many earlier studies did not provide convincing evidence that the lesions shown really were of acute traumatic origin. Traumatic white matter lesions may be confused with the nonspecific white matter high signal intensity foci associated with normal aging, hypertension [11,12] and affective disorders [19-21].

There are few earlier studies (field strengths from $0.064 \mathrm{~T}$ up to $1.5 \mathrm{~T}$ ) that selectively have investigated MR findings in concussion [5-9], and no study could be found in which exclusively the most common form of concussion has been studied, i.e. in those who had head traumas with LOC lasting maximally a few minutes. There were also inconsistent and inconclusive results as well as methodological shortcomings such as lack of or inadequate control group.

Taken the results and the mentioned methodological shortcomings of these earlier studies into account and in view of the results of the present study it seems justified to 
conclude that until now there is no reliable documentation that an ordinary concussion with short lasting LOC in a significant number of cases causes a degree of diffuse axonal injury that is visualized by conventional MR with a field strength up to $1.5 \mathrm{~T}$.

In recent years, several attempts have been made to detect signs of axonal injury by use of diffusion tensor imaging (DTI) MR, a method that is able to demonstrate white matter microstructure deficits

The findings in MTBI patients of these studies were usually discrete, inconsistent and diverging [22-28]. In one recent DTI tractography study of the corpus callosum in mild TBI 1 to 6 days post injury in 10 adolescents with LOC up to 10 minutes and 10 matched uninjured controls, fractional anisotrophy was increased and diffusivity decreased suggestive of cytotoxic edema, i.e. no sign of axonal injury [27]. None of the studies included subgroup analysis of patients with very short lasting LOC. As the size of the heterogeneous samples of patients with concussion generally was small, it is questionable whether any difference between findings in common concussion and controls would have reached statistical significance if only patients with short lasting LOC had been evaluated. An additional important source of error was that the included control groups usually consisted of non-injured healthy individuals. Since there is a link between alcohol consumption and injuries [29], concussion populations most likely have higher average alcohol consumption than non-injured controls. Alcohol abuse is, on the other hand, associated with white matter microstructure deficits shown by DTI in several regions of the brain, between others in areas prone to axonal injury after brain trauma [3033].

\section{Conclusion}

Using a rigidly controlled design with non-head injured controls and performing a baseline scan within 24 hours followed up with the same protocol at 3 months, this 1.0 T MR study shows that none of the patients with common concussion developed abnormalities that could be clinically (visually) detected and related to the brain trauma. Hence, no support was found for a potential organic substrate for PCS at this level of detection.

Obviously, the study does not rule out subtle traumatic abnormalities below the present detection threshold. In order to demonstrate such discrete traumatic changes (for example axonal injury) with conventional MR (or MR DTI) one should perform studies in a greater MTBI population than have been done hitherto and investigate only patients with common concussion (i.e., with LOC below 5 minutes), preferably using MR with a higher field strength (e.g., 3 T or more). Such studies should include a carefully matched control group of a sufficient size consisting of persons with a similar risk profile for MR changes, e.g. non-head injured individuals with alcohol consumption comparable to that of the MTBI group. Even if studies with this design should document traumatic lesions, it remains still uncertain whether lesions that are too discrete to be shown with a $1.0 \mathrm{~T}$ MR machine can explain the clinical symptoms of PCS.

\section{Competing interests}

The authors declare that they have no competing interests.

\section{Authors' contributions}

HS and DM conceived and designed the study, analyzed the data and drafted the manuscript. RG and SJ analyzed the MR and participated in the design and coordination of the study. DS participated in the study design and coordination and together with DM identified the patients in the study and control group. LJS participated in the design of the study, in analyzing the data and writing of the paper. DO participated in the design and coordination. All authors read and approved the final manuscript.

\section{Acknowledgements}

The work was carried out at the Emergency Unit, the Department of Neurology and the Department of Radiology of the Kaunas University of Medicine, Kaunas, Lithuania

\section{References}

I. Evans RW: The postconcussion syndrome and the sequelae of mild head injury. Neurol Clin 1992, I0(4):815-847.

2. Kiraly $M$, Kiraly S): Traumatic brain injury and delayed sequelae: a review - traumatic brain injury and mild traumatic brain injury (concussion) are precursors to later-onset brain disorders, including early-onset dementia. ScientificWorldjournal 2007, 7:1768-76.

3. Mittenberg W, DiGiulio DV, Perrin S, Bass AE: Symptoms following mild head injury: expectation as aetiology. J Neurol Neurosurg Psychiatry 1992, 55(3):200-204.

4. Ferrari R, Obelieniene D, Russell AS, Darlington P, Gervais R, Green $P$ : Symptom expectation after minor head injury. A comparative study between Canada and Lithuania. Clin Neurol Neurosurg 2001, I03(3): 184-190.

5. Doezema D, King JN, Tandberg D, Espinosa MC, Orrison WW: Magnetic resonance imaging in minor head injury. Annals of Emergency Medicine 1991, 20( I 2): I 281- I 285.

6. Voller B, Voller B, Benke T, Benedetto K, Schnider P, Auff E, Aichner F: Neuropsychological, MR and EEG findings after very mild traumatic brain injury. Brain Injury 1999, I3( I 0):82 I-827.

7. Hofman PA, Stapert SZ, van Kroonenburgh MJ, Jolles J, de Kruijk J, Wilmink JT: MR imaging, single-photon emission CT, and neurocognitive performance after mild traumatic brain injury. Am J Neuroradiol 200 I, 22(3):44 I-449.

8. Mittl RL, Grossman RI, Hiehle JF, Hurst RW, Kauder DR, Gennarelli TA, Alburger GW: Prevalence of MR evidence of diffuse axonal injury in patients with mild head injury and normal CT findings. Am J Neuroradiol 1994, I 5(8): I583-I598.

9. Topal NB, Hakyemez B, Erdogan C, Bulut M, Koksal O, Akkose S, Dogan S, Parlak M, Ozguc H, Korfali E: MR imaging in the detection of diffuse axonal injury with mild traumatic brain injury. Neurol Res 2008, 30/9:974-978.

10. Shaw NA: The neurophysiology of concussion. Prog Neurobiol 2002, 67(4):281-344.

II. Rao SM, Mittenberg W, Bernadin L, Haughton V, Leo GJ: Neuropsychological test findings in subjects with leukoaraiosis. Arch Neurol 1989, 46(I):40-44. 
12. Schmidt R, Fazekas F, Offenbacher H, Lytwyn H, Blematl B, Niederkorn K, Horner S, Payer F, Fredl W: Magnetic resonance imaging white matter lesions and cognitive impairment in hypertensive individuals. Arch Neurol I99I, 48(4):417-420.

13. Gentry LR, Godersky JC, Thompson B, Dunn VD: Prospective comparative study of intermediate-field $M R$ and $C T$ in the evaluation of closed head trauma. Am J Roentgenol 1988, 150(3):673-682.

14. Jin HQ, Araki S, Wu XK, Zhang YW, Yokoyama K: Psychological performance of accident-prone automobile drivers in China: a case-control study. Int J Epidemiol I99|, 20(I):230-233.

15. Poole GV, Lewis JL, Devidas M, Hauser C], Martin RW, Thomas KR Psychopathological risk factors for intentional and nonintentional injury. J Trauma 1997, 42(4):7II-7II5.

16. Lewine RR, Hudgins P, Brown F, Caudle J, Risch SC: Differences in qualitative brain morphology findings in schizophrenia, major depression, bipolar disorder, and normal volunteers. Schizophr Res 1995, 15(3):253-259.

17. Raine A, Lencz T, Bihrle S, LaCasse L, Colletti P: Reduced prefrontal gray matter volume and reduced autonomic activity in antisocial personality disorder. Arch Gen Psychiatry 2000, 57(2): II9-127.

18. Laakso MP, Vaurio O, Koivisto E, Savolainen L, Eronen M, Aronen H], Hakola P, Repo E, Soininen H, Tiihonen J: Psychopathy and the posterior hippocampus. Behav Brain Res 200I, I I8(2): I87-I93.

19. Coffey CE, Wilkinson WE, Weiner RD, Parashos IA, Djang WT, Webb MC, Figiel GS, Spritzer CE: Quantitative cerebral anatomy in depression. Arch Gen Psychiatry 1993, 50(1):7-16.

20. Dupont RM, Jemigan TL, Butters N, Delis D, Hesselink JR, Heindel W, Gillin JC: Subcortical abnormalities detected in bipolar affective disorder using magnetic resonance imaging. Arch Gen Psychiatry 1990, 47(I):55-59.

21. Dupont RM, Jemigan TL, Heindel W, Butters N, Shafer K, Wilson T, Hesselink J, Gillin JC: Magnetic resonance imaging and mood disorders. Arch Gen Psychiatry 1995, 52(9):747-755

22. Arfanakis K, Haughton VM, Carew JD, Rogers BP, Dempsey RJ, Meyerand ME: Diffusion tensor $M R$ imaging in diffuse axonal injury. Am J Neuroradiol 2002, 23(5):794-802.

23. Kraus MF, Susmaras T, Caughlin BP, Walker CJ, Sweeney JA, Little DM: White matter integrity and cognition in chronic traumatic brain injury: a diffusion tensor imaging study. Brain 2007, I 30(Pt I0):2508-25I9.

24. Bazarian J], Zhong J, Blyth B, Zhu T, Kavcic V, Peterson D: Diffusion tensor imaging detects clinically important axonal damage after mild traumatic brain injury: a pilot study. I Neurotraume 2007, 24(9): I 447-I 459

25. Benson RR, Meda SA, Vasudevan S, Kou Z, Govindarajan KA, Hanks RA, Millis SR, Makki M, Latif Z, Coplin W, Meythaler J, Haacke EM: Global white matter analysis of diffusion tensor images is predictive of injury severity in traumatic brain injury. J Neurotrauma 2007, 24(3):446-459.

26. Miles L, Grossman RI, Johnson G, Babb JS, Diller L, Inglese M: Shortterm DTI predictors of cognitive dysfunction in mild traumatic brain injury. Brain Injury 2008, 22(2): I I5-I22.

27. Wilde EA, McCauley SR, Hunter JV, Bigler ED, Chu Z, Wang ZJ, Hanten GR, Troyanskaya M, Yallampalli R, Li X, Chia J, Levin HS: Diffusion tensor imaging of acute mild traumatic brain injury in adolescents. Neurology 2008, 70(12):948-955.

28. Niogi SN, Mukherjee P, Ghajar J, Johnson CE, Kolster R, Lee H, Suh M, Zimmerman RD, Manley GT, McCandliss BD: Structural dissociation of attentional control and memory in adults with and without mild traumatic brain injury. Brain 2008, I3 I (Pt 1 2):3209-322I.

29. Nilsen $P$, Holmqvist $M$, Nordqvist $C$, Bendtsen P: Linking drinking to injury - causal attribution of injury to alcohol intake among patients in a Swedish emergency room. Int J Inj Contr Saf Promot 2007, 14(2):93-102.

30. Harris GJ, Jaffin SK, Hodge SM, Kennedy D, Caviness VS, Marinkovic K, Papadimitriou GM, Makris N, Oscar-Berman M: Frontal white matter and cingulum diffusion tensor imaging deficits in alcoholism. Alcohol Clin Exp Res 2008, 32(4): 1001 - I0I3.

31. De Bellis MD, Van Voorhees E, Hooper SR, Gibler N, Nelson L, Hege SG, Payne ME, MacFall J: Diffusion tensor measures of the corpus callosum in adolescents with adolescent onset alcohol use disorders. Alcohol Clin Exp Res 2008, 32(3):395-404.
32. Arnone D, Abou-Saleh MT, Barrick TR: Diffusion tensor imaging of the corpus callosum in addiction. Neuropsychobiology 2006, 54(2): $107-113$.

33. Pfefferbaum A, Adalsteinsson E, Sullivan EV: Dysmorphology and microstructural degradation of the corpus callosum: Interaction of age and alcoholism. Neurobiol Aging 2006, 27(7):994-1009.

\section{Pre-publication history}

The pre-publication history for this paper can be accessed here:

http://www.biomedcentral.com/1471-2342/9/11/prepub
Publish with Biomed Central and every scientist can read your work free of charge

"BioMed Central will be the most significant development for disseminating the results of biomedical research in our lifetime. "

Sir Paul Nurse, Cancer Research UK

Your research papers will be:

- available free of charge to the entire biomedical community

- peer reviewed and published immediately upon acceptance

- cited in PubMed and archived on PubMed Central

- yours - you keep the copyright
BioMedcentral 